\title{
Concept of Scalar-Vector Potential in the Contemporary Electrodynamic, Problem of Homopolar Induction and Its Solution
}

\author{
F.F. Mende* \\ B.I. Verkin Institute for Low Temperature Physics and Engineering NAS, Ukraine, 47 Lenin Ave., Kharkov, Ukraine \\ *Corresponding author: mende_fedor@mail.ru
}

Received September 26, 2014; Revised October 20, 2014; Accepted November 03, 2014

\begin{abstract}
At present classical electrodynamics consists of two not connected together parts. From one side this of Maxwell equations, which determine wave phenomena in the material media, from other side the Lorentz force, which determines power interaction between the moving charges. Still from the times of Lorenz and Poincare this force is introduced as experimental postulate. And as yet there is no that united basis, which connected together these two odd parts of the electrodynamics. Present article solves this problem on the basis of introduction the concept of scalar-vector potential, which assumes the dependence of the scalar potential of charge on its relative speed. In the article is carried out the analysis of the work of different of the schematics of the unipolar generators, among which there are diagrams, the principle of operation of which, until now, did not yield to explanation. The number of such diagrams includes the construction of the generator, whose cylindrical magnet, magnetized in the end direction, revolves together with the conducting disk. Postulate about the Lorentz force, whom is used for explaining the work of unipolar generators, does not give the possibility to explain the operating principle of this generator. It is shown that the concept of scalar- vector potential, developed by the author, gives the possibility to explain the operating principle of all existing types of unipolar generators. Physical explanation of Lorentz force in the concept of scalar- vector potential is given.
\end{abstract}

Keywords: laws of induction, electric field, scalar potential, magnetic field, vector potential, Maxwell equation, homopolar induction, unipolar generator

Cite This Article: F.F. Mende, "Concept of Scalar-Vector Potential in the Contemporary Electrodynamic, Problem of Homopolar Induction and Its Solution.” International Journal of Physics, vol. 2, no. 6 (2014): 202210. doi: 10.12691/ijp-2-6-4.

\section{Introduction}

Since Faraday opened the phenomenon of homopolar induction, past almost 200 years, but also up to now not all special features of this phenomenon found their explanation. Up to now unipolar generator is considered exception from the law of the induction of Faraday [1]. The attempts to explain all special features of homopolar induction with the aid of postulate about the Lorentz force did not give results. This postulate assumes that on the charge, which moves in the magnetic field, acts the force

$$
\vec{F}_{L}=e\left[\vec{v} \times \mu_{0} \vec{H}\right]
$$

In order to use this equation, it is necessary to know charge rate and must be assigned the external magnetic field, in which the charge moves. The oscillator circuit, which realizes the principle indicated, it is shown in Figure 1. Faraday also revealed that during the rotation of the conducting disk, magnetized in the end direction, on the brushes, which slide along the axis of disk and his generatrix, appears the electromotive force. This version of unipolar generator it is not possible to explain the aid of postulate about the Lorentz force.

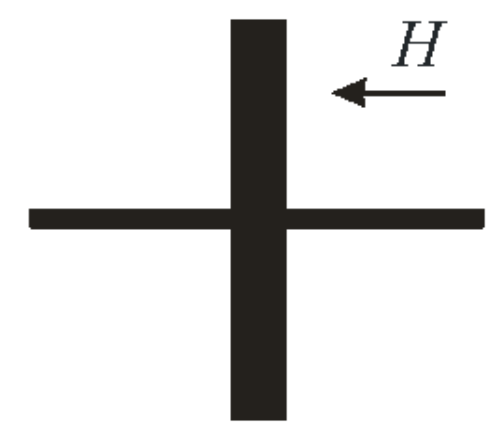

Figure 1. Unipolar generator, with the external magnetic field

During the rotation in the magnetic field of the rotor, made from conductor, free charges revolve together with the body of rotor, and Lorentz force acts on them, and the electromotive force appears between the axis of rotor and its periphery. The schematic of the unipolar generator, whose work cannot be explained with the aid of the postulate about the Lorentz force, is represented in Figure 2. 


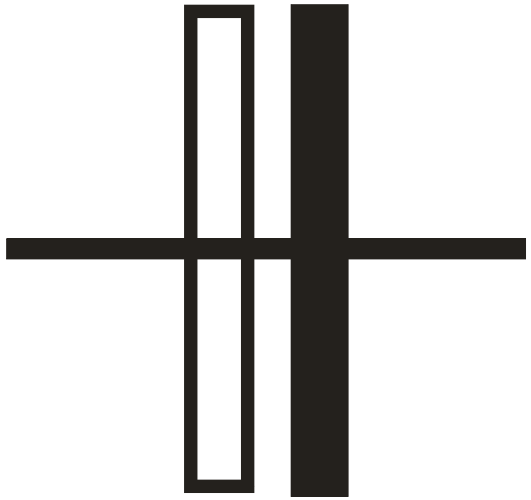

Figure 2. Unipolar generator with two disks

On the common axis are located two disks, one of which is magnetized, but no another. When both disks accomplish joint rotation, the electromotive force appears between the cheeks, which slide along the axis of the conducting disk and its generatrix. The electromotive force of the same value appears and the when conducting disk revolves, and the magnetized disk is fixed. It does not succeed to explain the work of this generator for that reason, that physical nature of very Lorentz force is not clear, and up to now it is introduced by axiomatic method. Therefore by the first task, which should be solved in order to explain the work of unipolar generators, the explanation of physical nature of Lorentz force appears.

\section{The phenomenon of the Lorentz force and its explanation in the concept of a scalar-vector potential}

\subsection{Introduction}

Laws of theclassical electrodynamics they reflect experimental facts they are phenomenological. Unfortunately, contemporary classical electrodynamics is not deprived of the contradictions, which did not up to now obtain their explanation.

The fundamental equations of contemporary classical electrodynamics are Maksvell equations. They are written as follows for the vacuum [2]

$$
\begin{array}{r}
\operatorname{rot} \vec{E}=-\frac{\partial \vec{B}}{\partial t} \\
\operatorname{rot} \vec{H}=\frac{\partial \vec{D}}{\partial t} \\
\operatorname{div} \vec{D}=0 \\
\operatorname{div} \vec{B}=0
\end{array}
$$

where $\vec{E}$ and $\vec{H}$ is tension of electrical and magnetic field, $\vec{D}=\varepsilon_{0} \vec{E}$ and $\vec{B}=\mu_{0} \vec{H}$ is electrical and magnetic induction, $\mu_{0}$ and $\varepsilon_{0}$ is magnetic and dielectric constant of vacuum. From these equations follow wave equations for the electrical and magnetic field

$$
\nabla^{2} \vec{E}=\mu_{0} \varepsilon_{0} \frac{\partial^{2} \vec{E}}{\partial t^{2}}
$$

$$
\nabla^{2} \vec{H}=\mu_{0} \varepsilon_{0} \frac{\partial^{2} \vec{H}}{\partial t^{2}}
$$

These equations show that in the vacuum can be extended the plane electromagnetic waves, the velocity of propagation of which is equal to the speed of light

$$
c=\frac{1}{\sqrt{\mu_{0} \varepsilon_{0}}}
$$

For the material media Maxwell equations they take the following form

$$
\begin{gathered}
\operatorname{rot} \vec{E}=-\mu \mu_{0} \frac{\partial \vec{H}}{\partial t}=-\frac{\partial \vec{B}}{\partial t} \\
\operatorname{rot} \vec{H}=n e \vec{v}+\varepsilon \varepsilon_{0} \frac{\partial \vec{E}}{\partial t}=n e \vec{v}+\frac{\partial \vec{D}}{\partial t} \\
\operatorname{div} \vec{D}=n e \\
\operatorname{div} \vec{B}=0
\end{gathered}
$$

where $\mu$ and $\varepsilon$ is the relative magnetic and dielectric constants of the medium and of $n, e$ and $\vec{v}$ is density, value and charge rate.

Of equations (2.1.1 -2.1.11) are written in the assigned inertial reference system (IRS), and in them there are no rules of passage of one IRS to another. Consequently, if are recorded wave equation in one IRS, then it is not known how to write down them in another frame of reference, which moves relative to the first system. The given equations also assume that the properties of charge do not depend on their speed, since in first term of the right side of equation (2.1.9) as the charge its static value is taken. These equations also assume that the current can leak both in the electrically neutral medium and to represent the isolated flow of the charged particles. These both situations are considered equivalent.

In Maksvell equations are not contained indication that is the reason for power interaction of the current carrying systems; therefore to be introduced the experimental postulate about the force, which acts on the moving charge in the magnetic field. This postulate assumes that on the charge, which moves in the magnetic field, acts the force [2]

$$
\vec{F}_{L}=e\left[\vec{v} \times \mu_{0} \vec{H}\right]
$$

However in this axiomatics is an essential deficiency. If force acts on the moving charge, then must be known the object, from side of which acts this force. In this case the magnetic field is independent substance, comes out in the role of the mediator between the moving charges. Consequently, there is no law of direct action, which would give answer to a question, as interact the charges, which accomplish relative motion.

Equation (2.1.12) causes bewilderment. In the mechanics the forces, which act on the moving body, are connected with its acceleration, with the uniform motion there exist frictional forces. The direction of these forces coincides with the velocity vector. But the force, determined by Eq. (2.1.12), have completely different property. Rectilinear motion causes the force, which is normal to the direction motion, what is assumed none of 
the existing laws of mechanics. Therefore is possible to assume that this some new law, which is concerned relative motion of those only of charged tel.

Is certain, magnetic field is one of the important concepts of contemporary electrodynamics. Its concept consists in the fact that around any moving charge appears the magnetic field (Ampere law), whose circulation is determined by the equation [2]

$$
\oint \vec{H} d \vec{l}=I
$$

where $I$ is conduction current. If we to the conduction current add bias current, then we will obtain the second equation of Maxwell (2.1.9).

It should be noted that the introduction of the concept of magnetic field does not be founded upon any physical basis, but it is the statement of the collection of some experimental facts, which with the aid of the specific mathematical procedures in large quantities of the cases give the possibility to obtain correct answer with the solution of practical problems. But there is a number of the physical questions, to which the concept of magnetic field answer does not give. Using Eqs. (2.1.12) and (2.1.13) not difficult to show that with the unidirectional parallel motion of two like charges, or flows of charges, between them must appear the additional attraction. However, if we pass into the inertial system, which moves together with the charges, then there magnetic field is absent, and there is no additional attraction. This paradox in the electrodynamics does not have an explanation. It does not have an explanation, also, in the special theory of relativity (STR).

With power interaction of conductors, along which flows the current, forces are applied not only to the moving charges, but to the lattice. But the concept of magnetic field also to this question answer does not give, since. In Eqs. (2.1.1-2.1.13) the presence of lattice is not considered. At the same time, with the flow of the current through the plasma its compression (pinch effect), occurs, in this case forces of compression act not only on the moving electrons, but also on the positively charged ions. And, again, the concept of magnetic field cannot explain this fact, since in this concept there are no forces, which can act on the ions of plasma.

As the fundamental law of induction in the electrodynamics is considered Faraday law, consequence of which is the first Maksvell equation. However, here are problems. It is considered Until now that the unipolar generator is an exception to the rule of flow. The existing state of affairs and those contradictions, which with this are connected, perhaps, are most are clearly formulated in the sixth volume of work [1]. We read on page 52 we read “..." flow rule”, according to which electromotive force in the outline it is equal to the speed undertaken with the opposite sign, with which changes magnetic flux through the outline, when flow changes due to field change or when outline moves (or when it occurs and that and, etc). Two of the possibility of - "the outline of moves" or "the field of changes" - are not distinguished of into of tuthe formulation of the rule. Nevertheless, for explaining the rule in these two cases we used two completely different laws: $[\vec{v} \times \vec{B}]$ for "moving outline" and $\nabla \times \vec{E}=-\frac{\partial \vec{B}}{\partial t}$ for “changing field”. "We know in physics not of one such example, if simple and precise general law required for its present understanding of analysis in the terms of two different phenomena. Usually of so beautiful of the generalization of proves to be of outgoing from of the united of the deep of that being basic of the principle of. But in this case of any separately deep principle it is not evident”.

All these examples be evidence the fact that the law of the induction of Faraday is inaccurate or not complete and does not reflect all possible versions of the appearance of electrical pour on with a change of the magnetic field or during the motion in the Ger.

From the aforesaid it is possible to conclude that physical nature of Lorentz force, which from the times of Lorenz and Poincare is introduced by axiomatic method, is not thus far known to us.

\subsection{Laws of the Induction}

With conducting of experiments established that in the outline is induced the current, when in the adjacent outline direct current is switched on or is turned off or adjacent outline with the direct current moves relative to the first outline. Therefore in general form Faraday law is written as follows [3]

$$
\oint \vec{E}^{\prime} d \vec{l}^{\prime}=-\frac{d \Phi_{B}}{d t}
$$

This writing of law indicates that with the determination of the circulation of $\vec{E}$ in the moving coordinate system, near $\vec{E}$ and $\vec{d} \vec{l}$ must stand primes and should be taken total derivative. But if circulation is determined in the fixed coordinate system, then primes near $\vec{E}$ and $d \vec{l}$ be absent, but in this case to the right in Eq. (2.2.1) must stand particular time derivative.

Complete time derivative in Eq. (2.2.1) indicates the independence of the eventual result of appearance electromotive force in the outline from the method of changing the flow. Flow can change as because $\vec{B}$ it depends on time, so also because the system, in which is determined the circulation $\oint \vec{E}^{\prime} d \vec{l}^{\prime}$, it moves in the magnetic field, whose value depends on coordinates. The value of magnetic flux in Eq. (2.2.1) is determined from the equation

$$
\Phi_{B}=\int \vec{B} d \vec{s}^{\prime}
$$

where the magnetic induction $\vec{B}=\mu \vec{H}$ is determined in the fixed coordinate system, and the element $d \vec{s}^{\prime}$ is determined in the moving system. Taking into account Eq. (2.2.1), we obtain from Eq. 2.(2.2)

$$
\oint \vec{E}^{\prime} d \vec{l}^{\prime}=-\frac{d}{d t} \int \vec{B} d \vec{s}^{\prime}
$$

since $\frac{d}{d t}=\frac{\partial}{\partial t}+\vec{v}$ grad let us write down $[4,5]$

$$
\oint \vec{E}^{\prime} d \vec{l}^{\prime}=-\int \frac{\partial \vec{B}}{\partial t} d \vec{s}^{\prime}-\int[\vec{B} \times \vec{v}] d \overrightarrow{l^{\prime}}-\int \vec{v} \operatorname{div} \vec{B} d \vec{s}^{\prime}
$$

In this case contour integral is taken on the outline $d \overrightarrow{l^{\prime}}$, which covers the area $d \vec{s}^{\prime}$. Let us immediately note that 
entire following presentation will be conducted under the assumption the validity of the Galileo conversions, i.e., $d \vec{l}^{\prime}=d l$ and $d \vec{s}^{\prime}=d \vec{s}$. During the motion in the magnetostatic field is fulfilled the

$$
\vec{E}^{\prime}=[\vec{v} \times \vec{B}] .
$$

Let us note that this equation is obtained not by the introduction of postulate about the Lorentz force. Thus, Lorentz force is the direct consequence of the law of magnetoelectric induction.

Faraday law indicates that how a change in the magnetic pour on, or motion in these fields, it leads to the appearance of electrical pour on; therefore it should be called the law of magnetoelectric induction. However, in the classical electrodynamics there is no law of electromagnetic induction, which would show, how a change in the electrical pour on, or motion in them, it leads to the appearance of magnetic pour on. The development of classical electrodynamics followed along another way. Was first known the Ampere law

$$
\oint \vec{H} d \vec{l}=I
$$

where $I$ is current, which crosses the area, included by the outline of integration. In the differential form Eq. (2.2.4) takes the form

$$
\operatorname{rot} \vec{H}=\vec{j}_{\sigma}
$$

where $\vec{j}_{\sigma}$ is current density of conductivity.

Maxwell supplemented Eq. (2.2.5) with bias current

$$
\operatorname{rot} \vec{H}=\vec{j}_{\sigma}+\frac{\partial \vec{D}}{\partial t}
$$

However, must exist the law of electromagnetic induction, which determines magnetic fields in the changing electric field

$$
\oint \vec{H}^{\prime} d \vec{l}^{\prime}=\frac{d \Phi_{D}}{d t}
$$

where $\Phi_{D}=\int \vec{D} d S^{\prime}$ is the flow of electrical induction $[4,5]$

$$
\oint \vec{H}^{\prime} d \overrightarrow{l^{\prime}}=\int \frac{\partial \vec{D}}{\partial t} d \vec{S}+\oint[\vec{D} \times \vec{V}] d \overrightarrow{l^{\prime}}+\int \vec{V} \operatorname{div} \vec{D} d \vec{S}^{\prime}
$$

In contrast to the magnetic pour on, when $\operatorname{div} \vec{B}=0$, for the electrical field on $\operatorname{div} \vec{D}=\rho$ and last term in the right side of Eq. (2.2.8) it gives the conduction current of $I$ and from Eq. (2.2.7) the Ampere law immediately follows. During the motion in the DC fields we obtain

$$
\vec{H}=[\vec{D} \times \vec{V}]
$$

As shown in the work [1], from Eq. (2.2.9) follows and Bio-Savart law, if for enumerating the magnetic pour on to take the electric fields of the moving charges. In this case the last member of the right side of Eq. (2.2.8) can be simply omitted, and the laws of induction acquire the completely symmetrical form [4,5,6,7]

$$
\begin{aligned}
& \oint \vec{E}^{\prime} d l^{\prime}=-\int \frac{\partial \vec{B}}{\partial t} d \vec{s}+\oint[\vec{v} \times \vec{B}] d l^{\prime} \\
& \oint \vec{H}^{\prime} d l^{\prime}=\int \frac{\partial \vec{D}}{\partial t} d \vec{s}-\oint[\vec{v} \times \vec{D}] d l^{\prime}
\end{aligned}
$$

For the constants fields on transformation laws they take the following form

$$
E^{\prime}=[\vec{v} \times \vec{B}], H^{\prime}=-[\vec{v} \times \vec{D}] .
$$

\subsection{Dynamic Potentials and the Field of the Moving Charges}

In the classical electrodynamics be absent the rule of the conversion of electrical and magnetic fields on upon transfer of one inertial system to another. This deficiency removes STR. With the entire mathematical validity of this approach the physical essence of such conversions up to now remains unexplained.

Let us explain, what potentials and fields can generate the moving charges. The first step, demonstrated in the works $[4,5,6,7]$, was made in this direction a way of the introduction of the symmetrical laws of magnetoelectric and electromagnetic induction Eq. (2.2.10).

Equations (2.2.10, 2.2.11) attest to the fact that in the case of relative motion of frame of references, between the fields $\vec{E}$ and $\vec{H}$ there is a cross coupling, i.e., motion in the fields $\vec{H}$ leads to the appearance fields $\vec{E}$ and vice versa. From these equations escape the additional consequences, which were for the first time examined in the work [4]. The electric field

$$
E=\frac{g}{2 \pi \varepsilon r}
$$

The electric field outside along charged rod decreases according to $\frac{1}{r}$.

If we in parallel to the axis of rod in the field of begin to move with the speed $\Delta v$ another IRS, then in it will appear the additional magnetic field. If we now with respect to already moving IRS, begin to move third frame of reference with the speed $\Delta v$, then already due to the motion in the field $\Delta H$ will appear additive to the electric field $\Delta E=\mu \varepsilon E(\Delta v)^{2}$. This process can be continued and further, as a result of which can be obtained the number, which gives the value of the electric field of $E_{v}^{\prime}(r)$ in moving IRS with reaching of the speed of $v=n \Delta v$, when $\Delta v \rightarrow 0$, and $n \rightarrow \infty$. In the final analysis in moving IRS the value of dynamic electric field will prove to be more than in the initial and to be determined by the equation

$$
E^{\prime}\left(r, v_{\perp}\right)=\frac{g c h \frac{v_{\perp}}{c}}{2 \pi \varepsilon r}=E c h \frac{v_{\perp}}{c}
$$

If speech goes about the electric field of the single charge, then its electric field will be determined by the equation 


$$
E^{\prime}\left(r, v_{\perp}\right)=\frac{e c h \frac{v_{\perp}}{c}}{4 \pi \varepsilon r^{2}}
$$

where $v_{\perp}$ is normal component of charge rate to the vector, which connects the moving charge and observation point.

Expression for the scalar potential, created by the moving charge, for this case will be written down as follows $[4,5,6,7]$

$$
\varphi^{\prime}\left(r, v_{\perp}\right)=\frac{e c h \frac{v_{\perp}}{c}}{4 \pi \varepsilon r}=\varphi(r) \operatorname{ch} \frac{v_{\perp}}{c}
$$

where $\varphi(r)$ is scalar potential of fixed charge. The potential $\varphi^{\prime}\left(r, v_{\perp}\right)$ can be named scalar-vector, since it depends not only on the absolute value of charge, but also on speed and direction of its motion with respect to the observation point. Maximum value this potential has in the direction normal to the motion of charge itself.

During the motion in the magnetic field, using the already examined method, we obtain

$$
H^{\prime}\left(v_{\perp}\right)=H \operatorname{ch} \frac{v_{\perp}}{c}
$$

where $v_{\perp}$ is speed normal to the direction of the magnetic field.

If we apply the obtained results to the electromagnetic wave and to designate components pour on parallel speeds IRSas $E_{\uparrow}, H_{\uparrow}$ and $E_{\perp}, H_{\perp}$ as components normal to it, then conversions pour on they will be written down

$$
\begin{aligned}
& \vec{E}_{\uparrow}^{\prime}=\vec{E}_{\uparrow}, \\
& \vec{E}_{\perp}^{\prime}=\vec{E}_{\perp} \operatorname{ch} \frac{v}{c}+\frac{Z_{0}}{v}\left[\vec{v} \times \vec{H}_{\perp}\right] \operatorname{sh} \frac{v}{c}, \\
& \vec{H}_{\uparrow}^{\prime}=\vec{H}_{\uparrow}, \\
& \vec{H}_{\perp}^{\prime}=\vec{H}_{\perp} \operatorname{ch} \frac{v}{c}-\frac{1}{v Z_{0}}\left[\vec{v} \times \vec{E}_{\perp}\right] \operatorname{sh} \frac{v}{c},
\end{aligned}
$$

where $Z_{0}=\sqrt{\frac{\mu_{0}}{\varepsilon_{0}}}$ is impedance of free space, $c=\sqrt{\frac{1}{\mu_{0} \varepsilon_{0}}}$ is speed of light.

Conversions fields (2.3.3) they were for the first time obtained in the work [4].

\subsection{Power Interaction of Parallel Conductors.}

The concept of magnetic field arose to a considerable degree because of the observations of power interaction of the current carrying and magnetized systems. Experience with the iron shavings, which are erected near the magnet poles or around the annular turn with the current into the clear geometric figures, is especially significant. These figures served as occasion for the introduction of this concept as the lines of force of magnetic field. In accordance with third Newton law with any power interaction there is always a equality of effective forces and opposition, and also always there are those elements of the system, to which these forces are applied. A large drawback in the concept of magnetic field is the fact that it does not give answer to that, counteracting forces are concretely applied to what, since. magnetic field comes out as the independent substance, with which occurs interaction of the moving charges.

Is experimentally known that the forces of interaction in the current carrying systems are applied to those conductors, whose moving charges create magnetic field. However, in the existing concept of power interaction of the current carrying systems, based on the concepts of magnetic field and Lorentz force, the positively charged lattice, which is the frame of conductor and to which are applied the forces, it does not participate in the formation of the forces of interaction.

Let us examine this question on the basis of the concept of scalar-vector potential $[4,5,6,7]$. We will consider that the scalar- vector potential of single charge is determined by Eq. (2.3.2), and that the electric fields, created by this potential, act on all surrounding charges, including to the charges positively charged lattices.

Let us examine from these positions power interaction between two parallel conductors (Figure 3), along which flow the currents. We will consider that $g_{1}{ }^{+}, g_{2}{ }^{+}$and $g_{1}{ }^{-}, g_{2}{ }^{-}$present positive and negative linear charges in the upper and lower conductors.

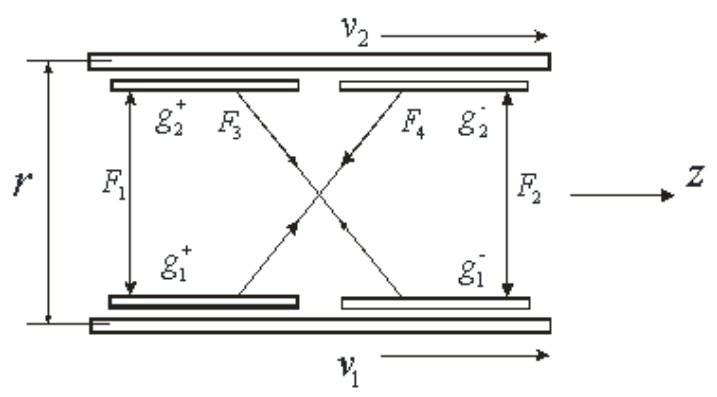

Figure 3. chematic of power interaction of the current carrying wires of two-wire circuit taking into account the positively charged lattice.

We will also consider that both conductors prior to the start of charges are electrically neutral, i.e., in the conductors there are two systems of the mutually inserted opposite charges with the specific density to, and which electrically neutralize each other. in Figure 1 these systems are moved apart along the axis $z$. Subsystems with the negative charge (electrons) can move with the speeds $v_{1}$ and $v_{2}$. The force of interaction between the lower and upper conductors we will search for as the sum of four forces, whose designation is understandable from the figure. The repulsive forces $F_{1}$ and $F_{2}$ we will take with the minus sign, while the attracting force $F_{3}$ and $F_{4}$ we will take with the plus sign.

For the single section of the two-wire circuit of force, acting between the separate subsystems, will be written down

$$
\begin{aligned}
& F_{1}=-\frac{g_{1}^{+} g_{2}^{+}}{2 \pi \varepsilon r}, F_{2}=-\frac{g_{1}{ }^{-} g_{2}{ }^{-}}{2 \pi \varepsilon r} \operatorname{ch} \frac{v_{1}-v_{2}}{c}, \\
& F_{3}=+\frac{g_{1}{ }^{-} g_{2}{ }^{+}}{2 \pi \varepsilon r} \operatorname{ch} \frac{v_{1}}{c}, F_{4}=+\frac{g_{1}{ }^{+} g_{2}{ }^{-}}{2 \pi \varepsilon r} \operatorname{ch} \frac{v_{2}}{c} .
\end{aligned}
$$

Adding forces, we will obtain the amount of the composite force 


$$
F_{\Sigma}=\frac{g_{1} g_{2}}{2 \pi \varepsilon r}\left(\operatorname{ch} \frac{v_{1}}{c}+\operatorname{ch} \frac{v_{2}}{c}-\operatorname{ch} \frac{v_{1}-v_{2}}{c}-1\right)
$$

In this expression as $g_{1}$ and $g_{2}$ are undertaken the absolute values of charges, and the signs of forces are taken into account in the bracketed expression. For the case $v<<c$ let us take only two first members of expansion in the series $\operatorname{ch} \frac{v}{c}$, i.e. we will consider that $\operatorname{ch} \frac{v}{c} \cong 1+\frac{1}{2} \frac{v^{2}}{c^{2}}$. From Eq. (2.4.2) we obtain

$$
F_{\Sigma 1}=\frac{g_{1} v_{1} g_{2} v_{2}}{2 \pi \varepsilon c^{2} r}=\frac{I_{1} I_{2}}{2 \pi \varepsilon c^{2} r}
$$

where $g_{1}$ and $g_{2}$ are undertaken the absolute values of specific charges, and $v_{1}$ and $v_{2}$ take with its signs.

Since the magnetic field of straight wire, along which flows the current $I$, we determine by the equation

$$
H=\frac{I}{2 \pi r}
$$

from Eq. (2.4.3) we obtain

$$
F_{\Sigma 1}=\frac{I_{1} I_{2}}{2 \pi \varepsilon c^{2} r}=\frac{H_{1} I_{2}}{\varepsilon c^{2}}=I_{2} \mu H_{1},
$$

where $H_{1}$ is the magnetic field, created by lower conductor in the location of upper conductor.

It is analogous

$$
F_{\Sigma 1}=I_{1} \mu H_{2}
$$

where $\mathrm{H}_{2}$ is the magnetic field, created by upper conductor in the region of the arrangement of lower conductor.

The results, obtained in the model of scalar- vector potential, completely coincide with the results, obtained on the basis of the concept of magnetic field.

Equation (2.4.3) represents the known rule of power interaction of the current carrying systems, but is obtained it not by the phenomenological way on the basis of the introduction of phenomenological magnetic field, but on the basis of completely intelligible physical procedures, under the assumption that that the scalar potential of charge depends on speed. In the formation of the forces of interaction in this case the lattice takes direct part, which is not in the model of magnetic field. In the model examined are well visible the places of application of force. The obtained equations coincide with the results, obtained on the basis of the concept of magnetic field and by the axiomatically introduced Lorentz force. In this case is undertaken only first member of expansion in the series $\operatorname{ch} \frac{v}{c}$. For the speeds $v \sim c$ should be taken all terms of expansion. In terms of this the proposed method is differed from the method of calculation of power interactions by the basis of the concept of magnetic field. If we consider this circumstance, then the connection between the forces of interaction and the charge rates proves to be nonlinear. This, in particular it leads to the fact that the law of power interaction of the current carrying systems is asymmetric. With the identical values of currents, but with their different directions, the attracting forces and repulsion become unequal. Repulsive forces prove to be greater than attracting force.

This difference is small and is determined by the equation

$$
\Delta F=\frac{v^{2}}{2 c^{2}} \frac{I_{1} I_{2}}{2 \pi \varepsilon c^{2} \varepsilon} .
$$

But with the speeds of the charge carriers of close ones to the speed of light it can prove to be completely perceptible.

Let us remove the lattice of upper conductor and after leaving only free electronic flux. In this case will disappear the forces $F_{1}$ and $F_{3}$, and this will indicate interaction of lower conductor with the flow of the free electrons, which move with the speed $v_{2}$ on the spot of the arrangement of upper conductor. In this case the value of the force of interaction is defined as

$$
F_{\Sigma}=\frac{g_{1} g_{2}}{2 \pi \varepsilon r}\left(\operatorname{ch} \frac{v_{2}}{c}-\operatorname{ch} \frac{v_{1}-v_{2}}{c}\right)
$$

The Lorentz force assumes linear dependence between the force, which acts on the charge, which moves in the magnetic field, and his speed. However, in the obtained equation the dependence of the amount of force from the speed of electronic flux will be nonlinear. From Eq. (2.4.4) of it is not difficult to see that with an increase in $v_{2}$ the deviation from the linear law increases, and in the case, when $v_{2}>>v_{1}$, the force of interaction are approached zero. This is very meaningful result. Specifically, this phenomenon observed in their known experiments Thompson and Kauffmann, when they noted that with an increase in the velocity of electron beam it is more badly slanted by magnetic field. They connected the results of their observations with an increase in the mass of electron. As we see reason here another.

Let us note still one interesting result. Taking into account Eq. (4.3), the force of interaction of electronic flux with the rectilinear conductor can be determined from the equation

$$
F_{\Sigma}=\frac{g_{1} g_{2}}{2 \pi \varepsilon r}\left(\frac{v_{1} v_{2}}{c^{2}}-\frac{1}{2} \frac{v_{1}^{2}}{c^{2}}\right) .
$$

From Eq. (2.4.5) follows that with the unidirectional electron motion in the conductor and in the electronic flux the force of interaction with the fulfillment of conditions of $v_{1}=\frac{1}{2} v_{2}$ is absent.

Since the speed of the electronic flux usually much higher than speed of current carriers in the conductor, the second term in the brackets in Eq. (2.4.5) can be disregarded. Then, since

$$
H_{1}=\frac{g_{1} v_{1}}{2 \pi \varepsilon c^{2} r}
$$

will the magnetic field, created by lower conductor in the place of the motion of electronic flux 


$$
F_{\Sigma}=\frac{g_{1} g_{2}}{2 \pi \varepsilon r} \frac{v_{1} v_{2}}{c^{2}}=g_{2} \mu v_{2} H
$$

In this case, the obtained value of force exactly coincides with the value of Lorentz force.

Taking into account that

$$
F_{\Sigma}=g_{2} E=g_{2} \mu v_{2} H
$$

it is possible to consider that on the charge, which moves in the magnetic field, acts the electric field $E$, directed normal to the direction of the motion of charge. This result also with an accuracy to of the quadratic terms of $\frac{v^{2}}{c^{2}}$ completely coincides with the results of the concept of magnetic field and is determined the Lorentz force, which acts from the side of magnetic field to the flow of the moving electrons.

As was already said, one of the important contradictions to the concept of magnetic field is the fact that two parallel beams of the like charges, which are moved with the identical speed in one direction, must be attracted. In this model there is no this contradiction already. If we consider that the charge rates in the upper and lower wire will be equal, and lattice is absent, i.e., to leave only electronic fluxes, then will remain only the repulsive force $F_{2}$.

Thus, the moving electronic flux interacts simultaneously both with the moving electrons in the lower wire and with its lattice, and the sum of these forces of interaction it is called Lorentz force. This force acts on the moving electron stream.

Regularly does appear a question, and does create magnetic field most moving electron stream of in the absence compensating charges? The diagram examined shows that the effect of power interaction between the current carrying systems requires in the required order of the presence of the positively charged lattice. Therefore most moving electronic flux cannot create that effect, which is created during its motion in the positively charged lattice.

\section{Operating Principle of Different Constructions of the Unipolar Generators}

In the previous division it was shown that the Lorentz force is the result of the dependence of the scalar potential of charge on the speed. Consequently, and the special feature of the work of different constructions of unipolar generators one should also search for by this method.

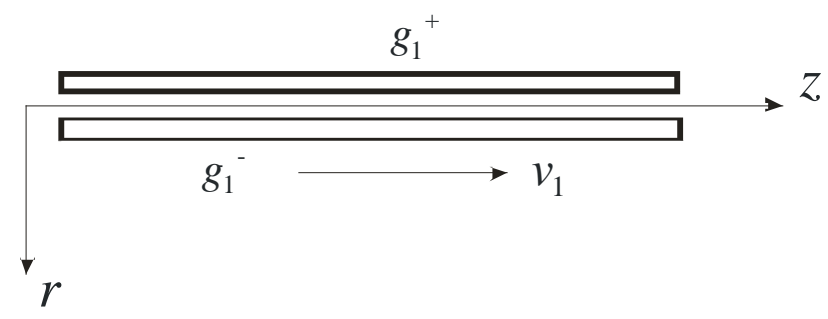

Figure 4. Section is the conductor, along which flows the current

Let us examine the case, when there is a single long conductor, along which flows the current. We will as before consider that in the conductor is a system of the mutually inserted charges of the positive lattice of $g^{+}$and free electrons of $g^{-}$, which in the absence current neutralize each other (Figure 4).

The electric field of conductor, created by rigid lattice, is determined by the equation

$$
E^{+}=\frac{g^{+}}{2 \pi \varepsilon r}
$$

We will consider that the direction of the vector of electric field coincides with the direction of $\vec{r}$. If the charges of electronic flux move with the speed $v_{1}$, then electrical field of flow is determined by the equation

$$
E^{-}=-\frac{g^{-}}{2 \pi \varepsilon r} \operatorname{ch} \frac{v_{1}}{c} \simeq-\frac{g^{-}}{2 \pi \varepsilon r}\left(1+\frac{1}{2} \frac{v_{1}^{2}}{c^{2}}\right)
$$

Adding Eq. (4.1) and Eq.(4.2), we obtain:

$$
E^{-}=-\frac{g^{-} v_{1}^{2}}{4 \pi \varepsilon c^{2} r}
$$

This means that around the conductor with the current is an electric field, which corresponds to the negative charge of conductor. However, this field has insignificant value, since in the real conductors $v \ll c$. This field can be discovered only with the current densities, which can be achieved in the superconductors, which is experimentally confirmed in papers $[8,9,10]$.

Let us examine the case, when very section of the conductor, on which with the speed $v_{1}$ flow the electrons, moves in the opposite direction with speed $v$ (Figure 5 . In this case Eqs. (4.1) and (4.2) will take the form

$$
\begin{gathered}
E^{+}=\frac{g^{+}}{2 \pi \varepsilon r}\left(1+\frac{1}{2} \frac{v^{2}}{c^{2}}\right) \\
E^{-}=-\frac{g^{-}}{2 \pi \varepsilon r}\left(1+\frac{1}{2} \frac{\left(v_{1}-v\right)^{2}}{c^{2}}\right)
\end{gathered}
$$

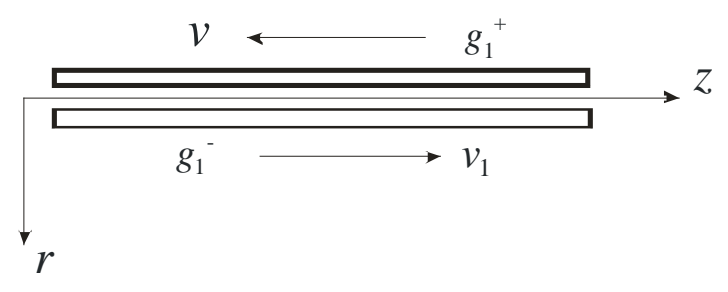

Figure 5. Conductor with the current, moving along the axis $\mathrm{Z}$

Adding Eqs. (4.3) and (4.4), we obtain

$$
E^{+}=\frac{g}{2 \pi \varepsilon r}\left(\frac{v v_{1}}{c^{2}}-\frac{1}{2} \frac{v_{1}^{2}}{c^{2}}\right)
$$

In this equation as the specific charge is undertaken its absolute value. Since the speed of the mechanical motion of conductor is considerably more than the drift velocity of electrons, the second term in the brackets can be disregarded 


$$
E^{+}=\frac{g v v_{1}}{2 \pi \varepsilon c^{2} r}
$$

The obtained result means that around the moving conductor, along which flows the current, is formed electric field. This is equivalent to appearance on the conductor of the linear positive charge of

$$
g^{+}=\frac{g v v_{1}}{c^{2}}
$$

If we conductor roll up into the ring and to revolve it then so that the linear speed of its parts would be equal $v$, then around this ring will appear the electric field, which corresponds to the presence on the ring of the specific charge indicated. But this means that the revolving turn, acquires electric charge. During the motion of linear conductor with the current the electric field will be observed with respect to the fixed observer, but if observer will move together with the conductor, then such fields will be absent. But if observer will move together with the conductor, then field will be absent for this observer.

In Figure 6. it is shown, as is obtained a voltage drop across the fixed contacts, which slide on the generatrix of the moving metallic plate, which is located near the moving conductor, along which flows the current.

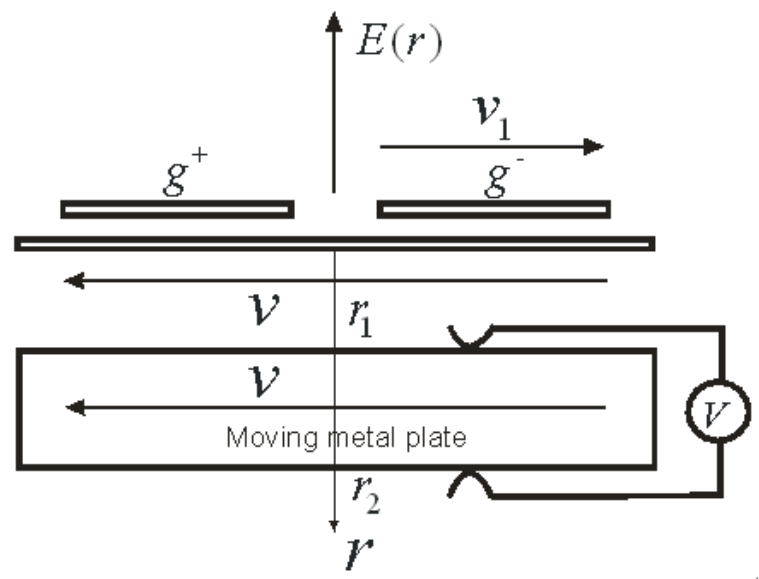

Figure 6. Diagram of the formation of the electromotive force of homopolar induction

We will consider that $r_{1}$ and $r_{2}$ of the coordinate of the points of contact of the tangency of the fixed contacts, which slide on the generatrix of metallic plate. Plate itself moves with the same speed also in the same direction as the conductor, along which flows the current. Contacts are connected to the voltmeter, which is also fixed. Then, it is possible to calculate a potential difference between these contacts, after integrating Eq. (4.6)

$$
U=\frac{g v v_{1}}{2 \pi \varepsilon c^{2}} \int_{r_{1}}^{r_{2}} \frac{d r}{r}=\frac{g v v_{1}}{2 \pi \varepsilon c^{2}} \ln \frac{r_{2}}{r_{1}}
$$

In order to apply to the contacts this potential difference, it is necessary sliding contacts to lock by the cross connection, on which a potential difference is absent. But since metallic plate moves together with the conductor, a potential difference is absent on it. It serves as the cross connection, which gives the possibility to convert this composite outline into the source of the electromotive strength, which acts in the circuit of voltmeter.
Now it is possible wire to roll up into the ring (Figure 7) and to feed it from the source of direct current. Instead of the single turn it is possible to use a solenoid. Contacts 1 should be connected to the collector ring, located on the rotational axis, and to the collector joined feeder brushes. Thus, obtain the revolving magnet. In this magnet should be placed the conducting disk with the opening (Figure 7), that revolves together with the turns of magnet, and with the aid of the fixed contacts, that slides on the generatrix of disk, tax voltage on the voltmeter. As the limiting case it is possible to take continuous metallic disk and to connect sliding contacts to the generatrix of disk and its axis. Instead of the revolving turn with the current it is possible to take the disk, magnetized in the axial direction, which is equivalent to turn with the current, in this case the same effect will be obtained. In this case the same effect will be obtained.



Figure 7. Schematic of unipolar generator with the revolving turn with the current and the revolving conducting ring

This diagram corresponds to the construction of the generator, depicted in Figure 2, when the conducting and magnetized disks revolve with the identical speed. The given diagram explains the work of unipolar generator with the revolving magnetized disk, since the conducting and magnetized disk it is possible to combine in one conducting magnetized disk.

The work of generator with the fixed magnetized disk and by the revolving conducting disk describes the diagram, represented by Figure 8.

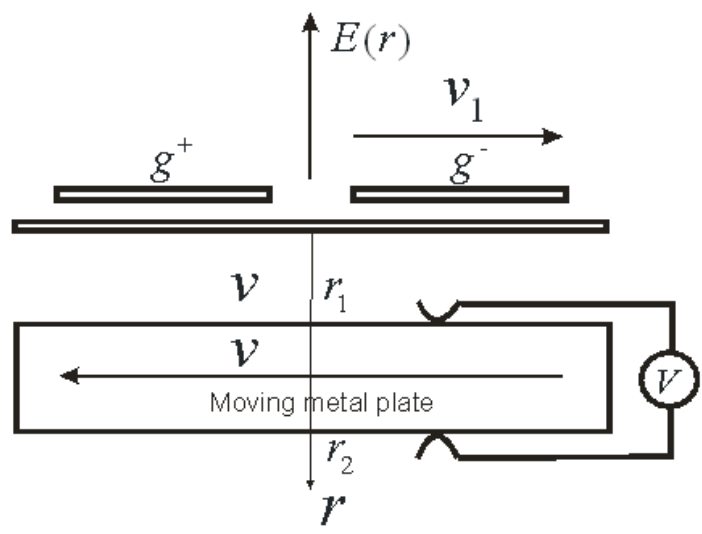

Figure 8. Equivalent the schematic of unipolar generator with the fixed magnet and the revolving conducting disk

In this case the following equations are fulfilled: 
The electric field, generated in the moving plate by the electrons, which move in the fixed conductor, is determined by the equation

$$
E^{-}=-\frac{g^{-}}{2 \pi \varepsilon r} \operatorname{ch} \frac{v_{1}-v}{c}=-\frac{g^{-}}{2 \pi \varepsilon r}\left(1+\frac{1}{2} \frac{\left(v_{1}-v\right)^{2}}{c^{2}}\right)
$$

and the electric field, generated in the moving plate by ions in the fixed conductor, is determined by the equation

$$
E^{+}=\frac{g^{+}}{2 \pi \varepsilon r} \operatorname{ch} \frac{v}{c}=\frac{g^{-}}{2 \pi \varepsilon r}\left(1+\frac{1}{2} \frac{v^{2}}{c^{2}}\right)
$$

The summary tension of electric field in this case will comprise

$$
E_{\Sigma}=\frac{g}{2 \pi \varepsilon r}\left(\frac{v v_{1}}{c^{2}}\right)
$$

The potential difference between the points $r_{1}$ and $r_{2}$ in the coordinate system, which moves together with the plate, we will obtain, after integrating this equation with respect to the coordinate

$$
U=\frac{g v v_{1}}{2 \pi \varepsilon c^{2}} \int_{r_{1}}^{r_{2}} \frac{d r}{r}=\frac{g v v_{1}}{2 \pi \varepsilon c^{2}} \ln \frac{r_{2}}{r_{1}}
$$

It is evident that this equation coincides with Eq. (4.7).

In the circuit of voltmeter, fixed with respect to the fixed conductor, a potential difference is absent; therefore the potential difference indicated will be equal to the electromotive force acting in the chain in question. As earlier moving conducting plate can be rolled up into the disk with the opening, and the wire, along which flows the current into the ring with the current, which is the equivalent of the magnet, magnetized in the end direction. Ring can be replaced with solenoid.

Thus, the concept of scalar-vector potential gives answers to all presented questions.

\section{Conclusion}

In the article is carried out the analysis of the work of different of the schematics of the unipolar generators, among which there are diagrams, the principle of operation of which, until now, did not yield to explanation. The number of such diagrams includes the construction of the generator, whose cylindrical magnet, magnetized in the end direction, revolves together with the conducting disk. Postulate about the Lorentz force, whom is used for explaining the work of unipolar generators, does not give the possibility to explain the operating principle of this generator. It is shown that the concept of scalar- vector potential, developed by the author, gives the possibility to explain the operating principle of all existing types of unipolar generators. Physical explanation of Lorentz force in the concept of scalar- vector potential is given.

\section{References}

[1] R. Feynman, R. Leighton, M. Sends, Feynman lectures on physics, M:, Mir, Vol. 6, 1977.

[2] V.V.Nicolsky, T.I. Nicolskaya, Electrodynamics and propagation of radio waves, Moscow, Nauka, 1989.

[3] J.Jackson, Classical Electrodynamics, Mir, Moscow, 1965.

[4] F.F. Mende, On refinement of equations of electromagnetic induction, Kharkov, deposited in VINITI, No 774 - B88 Dep., 1988.

[5] F.F. Mende, On refinement of certain laws of classical electrodynamics, arXiv.org/abs/physics/0402084.

[6] F. F. Mende, New electrodynamics. Revolution in the modern physics, Kharkov, NTMT, 2012.

[7] F. F.Mende, New approaches in contemporary classical electrodynamics, Part II, Engineering Physics, №2, 2013.

[8] W.F. Edwards, C.S. Kenyon, D.K. Lemon, Continuing investigation into possible electric arising from steady conduction current, Phys. Rev. D 14, 922 (1976).

[9] F.F. Mende, Experimental corroboration and theoretical interpretation of dependence of charge velocity on DC flow velocity through superconductors, Proceedings International Conference "Physics in Ukraine", Kiev 22-27 June, 1993.

[10] F. F. Mende, A New Tipe of Contact Potential Difference and Electrification of Superconducting Coils and Tori, American Journal of Electrical and Electronic Engineering, Vol. 2, No. 5, (2014), 146-151. 